\title{
Correction to: Preparation and Evaluation of Cubic Nanoparticles for Improved Transdermal Delivery of Propranolol Hydrochloride
}

\author{
Lingjun Zeng, ${ }^{1}$ Chun Tao, ${ }^{1}$ Zhihong Liu, ${ }^{1}$ Jing Zhang, ${ }^{1}$ Minxin Zhang, ${ }^{1}$ Jialiang Zhang, ${ }^{1}$ Shengnan Fang, ${ }^{1}$ \\ Xiaoyu Ma, ${ }^{2}$ Hongtao Song, ${ }^{1,3}$ and Xin Zhou ${ }^{1,3}$
}

published online 4 November 2020

Xin Zhou is a co-corresponding author of this published article and the affiliation should read "Xin Zhou", 3 ".

Correction to: AAPS PharmSciTech volume 21, Article number: 266 (2020) https://doi.org/10.1208/s12249-020-01809-7

Xin Zhou is a co-corresponding author of this published article and the affiliation should read "Xin Zhou", ${ }^{1,}$ ". Xin Zhou’s email address is fzzyyg@163.com.

The online version of the original article can be found at https:// doi.org/10.1208/s12249-020-01809-7

\footnotetext{
${ }^{1}$ Department of Pharmacy, Fuzong Clinical Medical College of Fujian Medical University (900 Hospital of the Joint Logistics Team), Fuzhou, 350025, People's Republic of China.

${ }^{2}$ College of Pharmacy, Fujian Medical University, Fuzhou, 350108, People's Republic of China.

${ }^{3}$ To whom correspondence should be addressed. (e-mail: shtfjfz@163.comfzzyyg@163.com)
} 